\title{
Challenges of transferring rural adults with chronic HCV infection for further HCV RNA confirmation and free DAAs treatment: a success story of the interdisciplinary collaboration approach
}

\author{
Wei Li ${ }^{1 \dagger}$, Te-Sheng Chang ${ }^{2+}$, Shu-Zhi Chang ${ }^{1}$, Ching-Hwa Chen ${ }^{3}$ and Mei-Yen Chen ${ }^{4,5,6^{*}}$ (0)
}

\begin{abstract}
Background: Chronic hepatitis C virus (HCV), which is a concern in many countries, is the leading cause of liver cancer around the world. Since Taiwan launched its national health insurance system in 1995, it has managed to extend health coverage to $99 \%$ of the Taiwanese population, providing free but limited antiviral treatment each year since 2017. However, many people in rural areas are unaware that they have chronic HCV; nor do they realize that new drugs with high cure rates could drastically reduce their health burden. The aim of this study is to explore the implementation facilitators of and barriers to inviting potentially infected patients in rural areas to be transferred for HCV ribonucleic acid (RNA) confirmation and new drug treatment.

Methods: A descriptive and prospective study design with an interdisciplinary collaboration approach was implemented. After five elements of referral were developed, telephone counseling was conducted between August 2018 and May 2019 in Yunlin, Taiwan. The elements of referral developed by the research team were: (1) forming and coordinating physicians' schedules, (2) recruiting and training volunteers, (3) training the nursing staff, (4) raising funds or resources, and (5) connecting with village leaders. Thereafter, we collaborated with two district health centers, a private local hospital, and health clinics. Based on the medical records provided by these agencies, community adults that were HCV antibody (anti-HCV) positive were invited to join the program.

Results: Of the 1795 adults who were serum anti-HCV positive, 1149 (64\%) accepted transfer to a qualified hospital; of these, 623 (54.2\%) had an HCV infection. 552 (88.6\%) of those infected started receiving direct-acting antivirals (DAAs) treatment. The top four barriers to accepting transfer were: (1) they perceived themselves to be healthy ( $n=98,32.3 \%)$; (2) mistrust of treatment/healthcare $(n=60,20.2 \%)$; (3) limited transportation to the hospital $(n=52$, (Continued on next page)
\end{abstract}

\footnotetext{
* Correspondence: meiyen@mail.cgust.edu.tw

'Wei Li and Te-Sheng Chang contributed equally to this work.

${ }^{4}$ Department of Nursing, Chang Gung University of Science and Technology, Chiayi, Taiwan

${ }^{5}$ Research Fellow, Department of Cardiology, Chang Gung Memorial Hospital, No. 2, Chiapu Rd. West Sec., Putz City, Chiayi County 613, Taiwan

Full list of author information is available at the end of the article
}

C C The Author(s). 2020 Open Access This article is licensed under a Creative Commons Attribution 4.0 International License, which permits use, sharing, adaptation, distribution and reproduction in any medium or format, as long as you give appropriate credit to the original author(s) and the source, provide a link to the Creative Commons licence, and indicate if changes were made. The images or other third party material in this article are included in the article's Creative Commons licence, unless indicated otherwise in a credit line to the material. If material is not included in the article's Creative Commons licence and your intended use is not permitted by statutory regulation or exceeds the permitted use, you will need to obtain permission directly from the copyright holder. To view a copy of this licence, visit http://creativecommons.org/licenses/by/4.0/. The Creative Commons Public Domain Dedication waiver (http://creativecommons.org/publicdomain/zero/1.0/) applies to the data made available in this article, unless otherwise stated in a credit line to the data. 
(Continued from previous page)
17.5\%); and (4) work conflict ( $n=30,10.1 \%)$.

Conclusion: An interdisciplinary collaboration approach significantly contributed to the invitation of CHC patients, as well as their acceptance of HCV RNA confirmation and free DAAs treatment. Using anti-HCV data from previous medical records for case-finding and collaborating with a hospital and health clinics proved to be an efficient strategy.

Keywords: Hepatitis C elimination, Anti-HCV positive, Direct-acting antivirals, Barriers, Facilitators, Rural

\section{Background}

According to the World Health Organization [1], more than 325 million people worldwide are chronically infected with hepatitis $\mathrm{B}$ or $\mathrm{C}$ virus ( $\mathrm{HBV}$ or $\mathrm{HCV}$ ). In many countries, mass infant vaccination has helped control HBV. However, no vaccine has been found to prevent $\mathrm{HCV}$ infection [2]. HCV is a blood-borne virus. For some people, it is a short-term illness; for more than $50 \%$ of people infected with $\mathrm{HCV}$, however, it is a longterm, chronic infection [2]. Chronic hepatitis $\mathrm{C}(\mathrm{CHC})$ is a concern in many countries, and is the leading cause of liver cirrhosis and hepatocellular carcinoma worldwide [3]. CHC, which is defined as being infected with HCV for more than 6 months, is responsible for $57 \%$ of cases of liver cirrhosis, $78 \%$ of cases of hepatocellular carcinoma, and an estimated 1.3 million preventable deaths a year worldwide [4]. Across the globe, approximately 71 million people are infected with $\mathrm{CHC}$ [5]. Research indicates that once a person is infected with $\mathrm{HCV}$, it becomes chronic in $75-85 \%$ of cases - with a high probability of progressing to liver cirrhosis and hepatocellular carcinoma [3]. Since the liver has no nerve endings and can function despite $70 \%$ of its mass being damaged, liver diseases are commonly referred to as "silent killers" and are often asymptomatic [4, 6].

The average prevalence rate of $\mathrm{HCV}$ infection in Taiwan is $3.87 \%$ [6]. The rate is even higher (10\%) in the country's southwestern coastal rural areas, making it significantly higher than those in the United States and European countries [1,7]. This phenomenon was initiated five decades ago when many rural residents of Taiwan were treated with inadequately disinfected medical equipment while sick. For instance, they were given injections by unqualified physicians, which caused many innocent people to be unknowingly infected by $\operatorname{HCV}[6,8]$. It is estimated that 400,000-700,000 people in Taiwan have $\mathrm{CHC}$. Patients with $\mathrm{CHC}$ have been treated with interferon-based therapies, which are commonly associated with adverse effects and long treatment duration [8]. Fortunately, the emergence of direct-acting antiviral (DAA) agents has proven to be a highly effective cure that also maintains patient safety [2, 6, 9-11]. The Taiwanese government has initiated a national elimination goal of eradicating $\mathrm{CHC}$ by 2025 , which will be paid for by the Taiwan National Health Insurance
(NHI). The free DAAs treatment was launched in 2017, with a maximum of around 11,000 treatments being provided annually, and each treatment costing 10,000 USD $[6,12]$.

Previous studies have indicated that universal $\mathrm{HCV}$ screening for adults, rather than just for birth cohorts and high-risk populations, is a key intervention tool used to inform people of their hepatitis status, and direct and motivate those infected to obtain the necessary medical care $[4,13,14]$. Given the costs, there are usually two steps to screen and confirm for an active HCV infection. The first step is to encourage people to participate in blood screening for anti-HCV; this is because being serum anti-HCV positive indicates either an active or a resolved $\mathrm{HCV}$ infection. If this test is positive, a confirmatory test-the HCV ribonucleic acid (RNA) test, also known as the $\mathrm{HCV}$ viral load test-is performed in order to identify active $\mathrm{CHC}$ infection $[1,15]$.

Although evidence indicates that DAA treatment for HCV has been successful, involves few side effects, and is free of charge through Taiwan's NHI $[6,8]$, many people with $\mathrm{HCV}$ do not have sufficient knowledge regarding liver health and treatment. For instance, Lin et al. [4] found that people who underwent blood screening for anti-HCV did not know the results nor their hepatitis status. Indeed, there is a gap that needs to be addressed in order to achieve the national elimination goal of eradicating $\mathrm{HCV}[4,14,16]$. In this regard, primary healthcare providers-who work at the frontline of the healthcare system-play an important role in actively promoting the free DAAs policy of the Taiwanese government. They should refer people with chronic HCV infections in rural areas for transfer to a qualified hospital for HCV RNA confirmation and free DAAs treatment. The aim of this study is to explore the implementation facilitators of and barriers to inviting potentially infected chronic $\mathrm{HCV}$ patients in rural areas to accept transfers for HCV RNA confirmation, and encouraging those who are HCV RNA positive to avail of free DAAs treatment.

\section{Methods}

\section{Design, sample, and setting}

A descriptive and prospective study design with an interdisciplinary collaboration approach was implemented between August 2018 and May 2019 in coastal Yunlin 
County. The researchers collaborated with a local hospital, three private outpatient clinics, and two district health centers that have been providing health screenings to community residents around the coastal western areas for over 10 years. All participants over 20 years old who were anti-HCV positive according to the medical records were invited to the collaborating hospital for further HCV RNA confirmation.

\section{Ethical considerations}

This study was approved by an ethical committee (Institutional Review Board No. 201701919B0) and was conducted in accordance with the principles of the Declaration of Helsinki (2008).

\section{Procedure}

The interdisciplinary collaboration approach, which involved five elements of referral and telephone counseling, was established by the research team as follows:

(1). Forming and coordinating physicians' schedules: Five gastroenterology and hepatology experts were invited to join the program and reach a consensus on $\mathrm{HCV}$ confirmation and the treatment protocol for people potentially infected with chronic HCV. The research team arranged five physicians' clinic schedules from Monday through Saturday based on the convenient outpatient time of the people invited for $\mathrm{HCV}$ RNA confirmation. Once HCV RNA positivity was confirmed, the patients would be persuaded by trained nursing staff to avail of the free DAAs treatment over a period of $8 \sim 12$ weeks, depending on one's genotype.

(2). Training the nursing staff to communicate four points during the telephone interview: Five nurses were trained to perform telephone interviews by emphasizing the following points: (a) treatment is provided for free: a free DAAs treatment saves patients at least 10,000 USD; (b) full understanding of the mechanism of HCV; (c) encouragement to join the national $\mathrm{HCV}$ elimination program; and (d) the reduction of transportation or transfer barriers. In addition, the nursing staff were trained to discuss the following: (a) who and where I am, (b) how I obtained your data, (c) why I called you, and (d) one call every other day during the first two weeks after $\mathrm{CHC}$ patients start treatment in order to make sure they take the oral drugs;

(3). Recruiting and training volunteers: Eight senior nursing students were trained to guide a resident when he or she was referred to a hospital for HCV confirmation and/or treatment;
(4). Raising funds or resources: Resources were collected, for example, for some poor families in order to resolve their transportation issues; and.

(5). Connecting with village leaders: The research team met with village leaders to ask them to publicize this project in all villages and assure community residents that a telephone call about the project would be from the research team and not a phone scam.

\section{Data analysis}

Microsoft Office Excel (2007) was used to record the demographic information of anti-HCV positive patients provided by the collaborating hospital or district health centers, such as the participants' names, gender, age, location, DAAs prescription, course of treatment, available times for telephone interview, and home telephone number. We also used Excel to record the participants' responses relating to their barriers to transferring to the hospital for HCV RNA confirmation. The participants' answers relating to barriers and facilitators regarding hospital referrals were divided into three main categories: (1) accepted referral confirmation, (2) refused referral confirmation, and (3) invalid calls or other factors. These were recorded as percentages by trained nursing staffs (Fig. 1).

\section{Results}

Of the 1795 adults who were serum anti-HCV positive according to the medical records of the collaborating hospital and clinics, 1149 (64\%) accepted a transfer to the hospital for HCV confirmation; among the 1149 patients who accepted transfer, 623 (54.2\%) were identified as being HCV RNA-positive (Fig. 2). Among them, 552 (88.6\%) were willing to undergo DAAs treatment for 23 months at the collaborating hospital, while 71 (11.4\%) were hesitant to do so (Fig. 3).

The finding shows the facilitators of the participants' willingness to be transferred in relation to the four main points communicated during the telephone interview: (a) the research team used "treatment is provided for free"

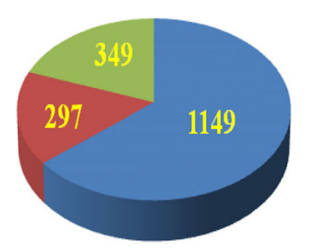

$$
\begin{aligned}
& \text { - accepted referral confirmation(64.0\%) } \\
& \text { refused referral confirmation(16.6\%) } \\
& \text { invalid calls or others(19.4\%) }
\end{aligned}
$$

Fig. 1 Anti-HCV positive $(n=1795)$ 


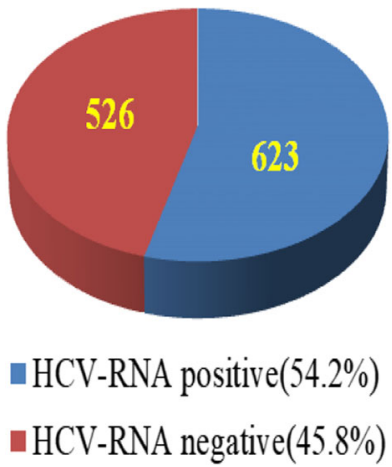

Fig. 2 Accept confirmation $(n=1149)$

to convince participants to accept the hospital referral since some residents had previously undergone treatment with interferon-based injections and experienced a lot of painful side effects because of it. Further, participants were introduced to the free oral medication (DAAs), which had a nearly $100 \%$ cure rate and relatively little side effects. A patient qualified for DAAs treatment can save up to 300,000 Taiwanese dollars (TWD) (equivalent to 10,000 USD). Many participants were happy because of this talk. Later, (b) "full understanding of the mechanism of HCV" was explained to the participants. For example, keeping in mind their health-related knowledge and social status, the participants were informed that a "virus like a worm" lives inside their body (liver). However, the virus cannot be easily detected as is it is asymptomatic and is very small; hence, the "worm" needs to be killed or "thrown out of the garden." During this step, many participants responded that they could understand the mechanism of $\mathrm{HCV}$ because of this talk.

Regarding (c) "let us join the national goal and take part in eradicating HCV," the participants were informed that the government initiated a free program. However, there are limitations as to the number of treatments provided yearly. Thus, the earlier the hepatitis is confirmed, the earlier treatment can be started; otherwise, it will be

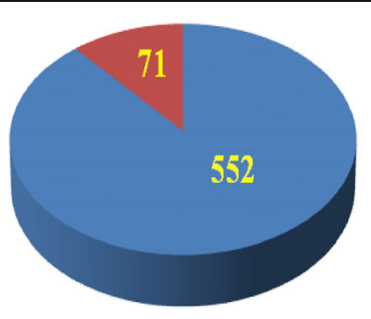

- adopted DAAs treatmet( $88.6 \%)$

- felt hesitation(11.4\%)

Fig. 3 HCV-RNA positive $(n=623)$ delayed to the next year. Many participants felt anxious during this step and reported barriers to their receipt of treatment. Regarding (d) "reducing transfer or treatment barriers," many rural residents mentioned that they faced barriers when transferring to another hospital, such as unfamiliarity with the hospital and transportation problems. The research team assured the participants that there would be someone waiting for them at the gate of the hospital, and that a volunteer (such as a village leader or nursing student) would guide them through each process at the hospital. In addition, the participants were informed that after they began the treatment, the research team would follow up on their response to the drugs. Many participants were reassured by this and immediately said yes.

Table 1 shows the barriers experienced by the 646 participants who did not confirm their treatment; 297 (46\%) participants directly refused to be transferred, while 349 (54\%) had an invalid telephone number, did not answer, or were dead or hospitalized. Among the 297 patients who refused confirmation, the top four barriers were as follows: (a) the self-perception that they were healthy $(n=98 ; 32.3 \%)$ (e.g., "I don't feel any discomfort at all! There is no need to see doctors! Just save the medical resources for others," "My liver doesn't feel painful, and I also need to work every day, so I don't have spare time to see doctors," "I don't think there is a need for me to see doctors; I do not know what hepatitis C is," "I don't feel any discomfort on a daily basis; it's really weird to take medicines for no reason"; (b) mistrust of treatment/ healthcare $((n=60 ; 20.2 \%)$ (e.g., "I am used to taking Chinese medicines instead of Western ones; I trust Chinese medicines more than Western ones"); (c)

Table 1 Barriers to transferring to a hospital for HCV RNA confirmation $(n=646)$

\begin{tabular}{lll}
\hline Variables & $\mathbf{N}$ & $\%$ \\
\hline Refusal to be referred for further confirmation $(n=297)$ & \\
Self-perception that they are healthy & 98 & 32.3 \\
Mistrust of treatment/healthcare & 60 & 20.2 \\
Transportation issues & 52 & 17.5 \\
Work conflict & 30 & 10.1 \\
Worried about the phone scam gang & 26 & 8.8 \\
Previously finished treatment & 22 & 7.4 \\
Difficulty listening & 8 & 2.7 \\
Poor perception of the hospital & 1 & 0.3 \\
Unable to contact ( $n=349)$ & & \\
No answer & 162 & 46.4 \\
Invalid telephone number & 142 & 40.7 \\
Death & 42 & 12.1 \\
Hospitalization & 3 & 0.8 \\
\hline
\end{tabular}


transportation issues $(n=52 ; 17.5 \%)$ (e.g., "I did not go to school when I was young; I don't even know how to take a shuttle bus to the hospital," and "The hospital is far away from my home ... it always takes me at least 2 hours to go see a doctor ... without my children's help, it's impossible for me to go to the hospital alone"); and (d) work conflict $(n=30 ; 10.1 \%)$ (e.g., "I need to work for my whole family; I have 3 work shifts," "It's impossible for me to take any time out to see a doctor; just wait and see ..., "I'm more worried about being jobless than having hepatitis," and "Without a job, I cannot afford a good life for my family").

\section{Discussion}

Although previous research has indicated that oral DAAs have a high cure rate over a short duration, have few side effects, and are free, many potential $\mathrm{HCV}$ patients still face barriers to viral detection and being referred for DAA treatment $[5,6,17]$. Therefore, the present study aimed to understand the implementation facilitators of and barriers to inviting potentially infected patients to accept transfer to a hospital for HCV RNA confirmation and free DAAs treatment in the western coastal Yunlin County [8]. Three key findings emerged from this study. First, it was an efficient strategy to use the anti-HCV data from the collaborating hospital's medical records for case-finding and to work with the collaborating hospital and clinics. Second, a nurse-led interdisciplinary collaboration involving five elements of referral and a telephone interview with four points for communication significantly facilitated the transfer of potential $\mathrm{CHC}$ patients to a hospital and increased their willingness to undergo DAAs treatment. Third, barriers to being referred to a hospital included a high percentage of patients who were unable to be contacted, the patients' self-perception of being healthy, mistrust of treatment/ healthcare, transportation issues, and work conflict.

Recently, in Australia, Pourmarzi et al. [18] demonstrated that a key to achieving the HCV elimination goal is the provision of its treatment in community settings, and that the integration and coordination of care and support provided for both patients and healthcare providers are important processes. The present study lends support to Pourmarzi et al. [18], who reported that successful elements of treatment in community settings include "training and support for healthcare providers, an open referral policy, linkage with or providing outreach services, a person-centered approach, and on-site screening and assessment." Further, to increase access to treatment for HCV in Australia, White et al. [19] developed a community-based FibroScan and a nurse-led service to assess patients. All patients had treatment prescribed and monitored in primary care, and a telephone follow-up was conducted to confirm that sustained virologic response (SVR) was performed by a clinic nurse. They found that the community-based model facilitates access to $\mathrm{HCV}$ treatment in primary care with excellent SVR rates. In Taiwan, the confirmation of HCV and prescription of DAA still requires specialty physicians. The authors of this study strongly recommend that community nurses or primary healthcare providers initiate an integrative program to increase public awareness and promote the referral system for HCV screening and treatment. For instance, there are 368 district public health centers in Taiwan, each with a mastery of the local population's annual health screening data. If these public institutions could duplicate the present study protocol-wherein once HCV RNA positivity was confirmed by the public health center, the patient would be persuaded to accept free DAAs treatment for $8 \sim 12$ weeks depending on his or her genotype-mobilizing such efforts to actively promote DAAs treatment would be an effective integrative program to eliminate $\mathrm{HCV}$ in Taiwan.

Unfortunately, many residents with anti-HCV refused to be referred to a hospital for further confirmation because they perceived themselves to be healthy, and appear to have no uncomfortable symptoms. This finding is similar to that of Cheng et al. [20], who conducted a study in remote southern Taiwan. They stated that less than half of confirmed $\mathrm{HCV}$-infected residents received adequate medical care. In addition, Treloar and her colleagues [16] described barriers to $\mathrm{HCV}$ care and stigmatization from a social perspective. Previous studies have emphasized the relationship between stigma and adverse health outcomes as well as health access measures. Stigma is a defining factor in $\mathrm{HCV}$ treatment, given the association of $\mathrm{HCV}$ with the socially demonized practice of injection drug use [16, 21]. Although the present study did not explore the effect of stigma on hospital transfers, it is necessary to comprehensively understand why some people refuse to be referred to a hospital. Nonetheless, when the present researchers approached the residents, some responded that they are worried about the phone scam gang, while others reported having transportation issues or not having enough time because of their need to keep working.

Regarding the barriers of hospital transfer, for rural residents, the hospital is $40-60 \mathrm{~km}$ away from the village, and it is particularly difficult for elders to get to without transportation assistance. In addition, it was hard for our research team to reach most patients to notify them because they do not have correct telephone numbers and many residents do not care about DAAs treatment because they feel healthy. Thus, the barriers to being referred to a hospital were clarified. The research team emphasized the communication of the following points to the anti-HCV positive villagers: (1) Since the Taiwanese 
government fully subsidizes DAAs treatment, patients should seize the opportunity to avail of the free treatment before the annual limit is reached; (2) health is priceless and is the most worthy investment; the sooner you complete your DAAs treatment, the sooner you can rid yourself of the threat of liver cancer; (3) with regard to the 552 patients concerned about adopting the treatment, appropriate health education was given as well as regular reminders to take their medicine; (4) weekly calls were made to remind the patients to return to the hospital for treatment completion; and (5) those who experienced excellent results after undergoing the DAAs treatment were encouraged to promote acceptance of treatment among other patients.

The findings from this study strongly suggest that to achieve the $\mathrm{HCV}$ elimination goal of eradicating $\mathrm{HCV}$ nationwide, there is a need to make vigorous efforts to enhance screening coverage, educate people about new information relating to liver disease, reduce the barrier of work conflict, and use the media to promote related policy and hospital referrals. The present findings showed a high percentage of invalid or disconnected home telephone numbers $(n=304)$. This may be due to wrong numbers being included in the medical records provided by the collaborating hospital and clinics. This could also be attributed to the fact that the patients' health screenings were conducted many years ago, and they changed their telephone numbers in recent years. Therefore, to increase case findings, it is crucial to collect correct information through multiple sources (e.g., ask for help from the district government, or visit village leaders and make home visits).

This study has some limitations that should be noted. First, although the research team encouraged all healthcare providers in the outpatient clinics to survey medical records and refer each anti-HCV patient to the collaborating hospital for $\mathrm{HCV}$ confirmation, some medical personnel from the private sector had reservations regarding this approach (e.g., some of them felt that it was not so important). In addition, there were no incentives for primary healthcare providers from public health centers to make transfer referrals. Consequently, this may limit the case findings from rural areas. Second, the high percentage of invalid telephone numbers and failure to answer the telephone limits the referrals and the effectivity of the approach used in the study. Thus, it is necessary to reestablish valid telephone connections via the public and private sectors. Third, Taiwan has launched the NHI system and covers almost all of the Taiwanese population. However, since each item for the antibody test costs less than 7 USD, while each HCV RNA confirmation costs over 70 USD, the government uses two steps to confirm an active HCV infection. This procedure increases the barriers to hospital transfer for HCV RNA confirmation and DAAs treatment of $\mathrm{CHC}$ patients. Therefore, we strongly suggest that confirmatory testing (HCV RNA) should be done locally, with referrals for those that are HCV RNA positive.

\section{Conclusion}

The findings of this study show that the interdisciplinary collaboration approach, with five elements of referral and a telephone interview wherein four points for communication are emphasized, significantly influenced the patients' acceptance of hospital referrals and their willingness to undergo a new drug treatment in endemic rural areas of Taiwan. In addition, it was an efficient strategy to use medical records provided by the collaborating hospital, clinics, and district health centers to identify residents who were anti-HCV positive and increase case findings. Future research should focus on reducing barriers to hospital transfer and treatment in disadvantaged areas, such as transportation issues, work shifts, invalid telephone numbers, and incorrect self-perceptions of being healthy.

\section{Abbreviations}

HCV: Hepatitis C virus; CHC: Chronic hepatitis C; anti-HCV: Hepatitis C virus antibody positive; DAA: Direct-acting antiviral agents

\section{Acknowledgements}

The authors would like to express our appreciation to all participants who participated in this study.

\section{Declarations}

The authors declare no conflict of interest.

\section{Authors' contributions}

Methodology, formal analysis, and writing —original draft preparation, review and editing, WL, MYC and TSC; investigation and data creation, SZC, and CHC; conceptualization, project administration, and supervision, MYC. All authors have read and approved the manuscript.

\section{Funding}

The study was supported by a grant from Taiwan Ministry of Science and Technology (MOST-107-2314-B-255-001-MY3) and Formosa Plastic Group (FCRP F610011). The funding bodies had no role in the design of the study and collection, analysis, and interpretation of data and in writing the manuscript.

\section{Availability of data and materials}

The datasets analyzed during the current study are not yet publicly available but are available from the corresponding author on reasonable request.

Ethics approval and consent to participate

All the subjects provided written informed consent before participating in the study, and the study design was approved by the Chang Gung Memorial Hospital's Institutional Review Board (No: 201701919B0).

\section{Consent for publication}

Not Applicable.

\section{Competing interests}

The authors declare no conflict of interest.

\section{Author details}

${ }^{1}$ Formosa Plastics Group Health Care, Yunlin, Taiwan. ${ }^{2}$ Department of Internal Medicine, Division of Gastroenterology and Hepatology, Chang Gung Memorial Hospital, Yunlin, Taiwan. ${ }^{3}$ Formosa Plastics Group, Advanced Engineer, Safety Health and Environment Center, Taipei, Taiwan.

${ }^{4}$ Department of Nursing, Chang Gung University of Science and Technology, Chiayi, Taiwan. ${ }^{5}$ Research Fellow, Department of Cardiology, Chang Gung 
Memorial Hospital, No. 2, Chiapu Rd. West Sec., Putz City, Chiayi County 613,

Taiwan. ${ }^{6}$ School of Nursing, Chang Gung University, Taoyuan, Taiwan.

Received: 12 April 2020 Accepted: 20 September 2020

Published online: 07 October 2020

\section{References}

1. World Health Organization, WHO. Fact sheet: Hepatitis C. http://www.who. int/news-room/fact-sheets/detail/hepatitis-c (accessed 30 August 2019).

2. Centers for Disease Control and Prevention, CDC. Viral hepatitis. https:// www.cdc.gov/hepatitis/ (accessed 1 August 2019).

3. Tada T, Toyoda H, Yasuda S, Miyake N, Kumada T, Kurisu A, et al. Natural history of liver-related disease in patients with chronic hepatitis $C$ virus infection: an analysis using a Markov chain model. J Med Virol. 2019;91(10): 1837-44.

4. Lin C, Clark R, Tu P, Tu R, Hsu YJ, Nien HC. The disconnect in hepatitis screening: participation rates, awareness of infection status, and treatmentseeking behavior. J Glob Health. 2019;9(1):010426.

5. Lanini S, Easterbrook PJ, Zumla A, Ippolito G. Hepatitis C: global epidemiology and strategies for control. Clin Microbiol Infect. 2016;22:833-8.

6. National Health Insurance, NHI. Hepatitis C full oral new drug health insurance payment implementation plan. https:/www.nhi.gov.tw/Content_ List.aspx?n=A4EFF6CD1C4891CA\&topn=3FC7D09599D25979 (accessed 10 August 2019).

7. Rosenberg ES, Rosenthal EM, Hall EW, Barker L, Hofmeister MG, Sullivan PS, et al. Prevalence of hepatitis C virus infection in US states and the District of Columbia, 2013 to 2016. JAMA Netw Open. 2018;1(8):e186371.

8. Taiwan Medical Association, TMA. Hepatitis C epidemiology and prevention strategy. http://www.fma.org.tw/2016/l-1-3.html (accessed 20 August 2019).

9. Domont $F$, Cacoub P. Chronic hepatitis $C$ virus infection, a new cardiovascular risk factor? Liver Int. 2016;36(5):621-7.

10. Yoo ER, Perumpail RB, Cholankeril G, Jayasekera CR, Ahmed A. Expanding treatment access for chronic hepatitis $C$ with task-shifting in the era of direct-acting antivirals. J. Clin. Transl. Hepatol. 2017;5(2):130-3.

11. Younossi ZM, Park H, Dieterich D, Saab S, Ahmed A, Gordon SC. The value of cure associated with treating treatment-naïve chronic hepatitis $C$ genotype 1: Are the new all-oral regimens good value to society? Liver Int. 2017;37(5):662-8.

12. Chen DS. Taiwan commits to eliminating hepatitis C in 2025. Lancet Infect. Dis. 2019;19(5):466

13. Saab S, Le L, Saggi S, Sundaram V, Tong MJ. Toward the elimination of hepatitis $C$ in the United States. Hepatology. 2018;67(6):2449-59.

14. Ward JW, Mermin JH. Simple, effective, but out of reach? Public health implications of HCV drugs. N. Engl J Med 2015;73(27):2678-80.

15. Castro R, Crathorne L, Perazzo H, Silva J, Cooper C, Varley-Campbell J, et al. Cost-effectiveness of diagnostic and therapeutic interventions for chronic hepatitis C: a systematic review of model-based analyses. BMC Med Res Methodol. 2018;18(1):53.

16. Treloar C, Rance J, Backmund M. Understanding barriers to hepatitis C virus care and stigmatization from a social perspective. Clin Infect Dis. 2013; 57(suppl2):S51-5.

17. Burki T. Eliminating hepatitis C. Lancet Infect Dis. 2019;19:246-7.

18. Pourmarzi D, Smirnov A, Hepworth J, Rahman T, FitzGerald G, Hall L. Elements of community-based models for treating hepatitis $C$ virus in supporting HCV elimination in Australia: A Delphi study. Int J Health Plann Manage. 2019;34(2):e1247-56

19. White L, Azzam A, Burrage L, Orme C, Kay B, Higgins S,O'Beirne J. Facilitating treatment of HCV in primary care in regional Australia: closing the access gap. Frontline Gastroenterol. 2019;10(3):210-6.

20. Cheng PN, Chiu YC, Chiu HC, Chien SC. The characteristics of residents with unawareness of hepatitis C virus infection in community. PloS One. 2018; 13(2):e0193251.

21. Butt G. Stigma in the context of hepatitis C: concept analysis. J Adv Nurs. 2008;62(6):712-24.

\section{Publisher's Note}

Springer Nature remains neutral with regard to jurisdictional claims in published maps and institutional affiliations.

\section{Ready to submit your research? Choose BMC and benefit from:}

- fast, convenient online submission

- thorough peer review by experienced researchers in your field

- rapid publication on acceptance

- support for research data, including large and complex data types

- gold Open Access which fosters wider collaboration and increased citations

- maximum visibility for your research: over $100 \mathrm{M}$ website views per year

At BMC, research is always in progress.

Learn more biomedcentral.com/submissions 